\title{
Global regulation of robots using only position measurements
}

\author{
Harry Berghuis \\ Department of Electrical Engineering, Mechatronics Research \\ Centre Twente, University of Twente, P.O. Box 217,7500 AE \\ Enschede, Netherlands
}

\section{Henk Nijmeijer}

Department of Applied Mathematics, Mechatronics Research Centre Twente, University of Twente, P.O. Box 217, 7500 AE Enschede, Netherlands

Received 28 November 1992

Revised 16 February 1993

\begin{abstract}
In this note we propose a simple solution to the regulation problem of rigid robots based on the availability of only joint position measurements. The controller consists of two parts: (1) a gravitation compensation, (2) a linear dynamic firstorder compensator. The gravitation compensation part can be chosen to be a function of either the actual joint position or the desired joint position. Both possibilities are proved to yield global asymptotic stability. Performance issues of the controller are illustrated in a simulation study of a two degrees-of-freedom robot manipulator.
\end{abstract}

Keywords: Robot control; dynamic compensator; Lyapunov theory.

\section{Introduction}

In the last decade, several strategies that solve the regulation (also known as position or pointto-point control) and tracking control problem have been presented in the literature, see for instance [14]. One main ingredient in the majority of these controllers is a proportional-derivative (PD) feedback, which shows that these controllers rely on the assumption of ideal full state information. Unfortunately, in practice this assumption can only partially be fulfilled for two reasons. First, although

Correspondence to: $\mathrm{H}$. Berghuis, Department of Electrical Engineering, Mechatronics Research Centre Twente, University of Twente, P.O. Box 217, 7500 AE Enschede, Netherlands. robot systems generally are equipped with highprecision sensors for position measurements, velocity measurements, for instance from tacho meters, are often contaminated with a considerable amount of noise. Second, in robotic applications today, velocity sensors are frequently omitted due to the considerable savings in cost, volume and weight that can be obtained in this way [8].

One way to remedy the velocity feedback problem is to determine a velocity signal by first-order numerical differentiation of the accurate position signal. The simplicity of this technique makes it particularly useful from an implementation point of view. However, especially for low- and high-range velocities, such a simple approximation of the velocity signal may be inadequate $[1,4]$. Moreover, the quantization effect that inherently goes along with this approach may produce undesired oscillations in the robot joint response, or even cause it to become unstable [8]. In addition, up to now there is no theoretical justification for this ad hoc solution, that is closed-loop stability properties have not been proved.

Motivated by the foregoing, recently the control problem of robots using only position measurements have attained an increasing interest. A straightforward approach to this problem goes along a two-step design procedure: (1) construct an observer, driven by the available inputs and outputs, which reconstructs the lacking velocity signal, (2) design a state-feedback controller and replace the actual velocity by the one reconstructed from the observer. Indeed, based on this procedure a number of conceptually different methods for both regulation and tracking control of robots equipped with only position sensors have been developed, see for instance $[2-5,10,11]$. Unfortunately, as opposed to the state-feedback solutions to robot control, the stability properties of these controller-observer combinations are only of local nature. This shows one important drawback of these controller-observer combinations. 
In this note we adopt a different approach that allows us to come up with a globally asymptotically stable solution to the regulation problem of robots using only position feedback. The starting point for our result is the beautifully simple position controller of Takegaki and Arimoto [15]. This controller is based on the intuitive idea of reshaping the potential energy of the robot system such that it achieves a global energy minimum at the desired equilibrium position, and add damping via velocity feedback for global asymptotic stabilization purposes. Our results also employ the energy reshaping idea, but we inject damping in the control loop via a first-order linear compensator, which requires only position measurements. Despite this modification, global asymptotic stability is preserved, as we will show. A simulation study of a two-degrees-offreedom robot manipulator supports the theoretical results.

The organization of this note is as follows. In Section 2 our main results are presented. Section 3 discusses the outcomes of a simulation study. We end with some concluding remarks.

\section{Global regulation using only position feedback}

\subsection{Main result}

Consider the general equations describing the dynamics of an $n$-degrees-of-freedom rigid robot manipulator

$$
M(q) \ddot{q}+C(q, \dot{q}) \dot{q}+G(q)=\tau,
$$

where $q$ is the $n \times 1$ vector of generalized coordinates and $\tau$ is the $n \times 1$ vector of external torques, $M(q)$ represents the $n \times n$ positive-definite inertia matrix, $C(q, \dot{q}) \dot{q}$ is the $n \times 1$ vector of Coriolis and centrifugal torques, and $G(q)$ is the $n \times 1$ vector of gravitational torques.

Before presenting our main result, let us recall the seminal paper by Takegaki and Arimoto [15] concerning the position control problem of robots. These authors propose the controller (amongst others)

$\tau=G(q)-K_{\mathrm{d}} \dot{q}-K_{\mathrm{p}} e$,

where $K_{\mathrm{d}}=K_{\mathrm{d}}^{\mathrm{T}}>0, K_{\mathrm{p}}=K_{\mathrm{p}}^{\mathrm{T}}>0$ and $e \equiv q-q_{\mathrm{d}}$ represents the position error, and $q_{\mathrm{d}}$ is the constant desired position. This controller consists of a gravitation compensation and a linear static state feed- back, which underscores its simplicity. To prove global asymptotic stability of the closed-loop dynamics ((2.1) and (2.2)), i.e.

$M(q) \ddot{q}+C(q, \dot{q}) \dot{q}+K_{\mathrm{d}} \dot{q}+K_{\mathrm{p}} e=0$,

in [15] the modified energy function

$V_{1}(\dot{q}, e)=\frac{1}{2} \dot{q}^{\mathrm{T}} M(q) \dot{q}+\frac{1}{2} e^{\mathrm{T}} K_{\mathrm{p}} e$

was used as Lyapunov function. The time-derivative of $V_{1}(\dot{q}, e)$ along the closed-loop dynamics (2.3) equals

$\dot{V}_{1}(\dot{q}, e)=-\dot{q}^{\mathrm{T}} K_{\mathrm{d}} \dot{q}$.

Although this time-derivative is only negativesemi-definite, global asymptotic stability can be established by invoking LaSalle's invariance theorem [6]. Characteristic of the controller (2.2) is that the gravitation compensation part together with the proportional feedback reshape the potential energy of the robot system, and that the velocity term injects damping in the loop (cf. [13]).

One practical disadvantage of the controller (2.2) is the need for velocity measurements. To overcome this problem, we propose to modify the controller (2.2) as

$$
\begin{aligned}
& \tau=G(q)-K_{\mathrm{d}} \dot{x}-K_{\mathrm{p}} e, \\
& \dot{x}=-L x+K_{\mathrm{d}} e,
\end{aligned}
$$

where $L=L^{\mathrm{T}}>0$. Then we can prove the following result.

Theorem 2.1. The position controller (2.6) globally asymptotically stabilizes the robot system (2.1) at the equilibrium point $(\dot{q}, e, x) \equiv 0$.

Proof. The equations for closed-loop error dynamics are

$$
\begin{aligned}
& M(q) \ddot{q}+C(q, \dot{q}) \dot{q}+K_{\mathrm{d}} \dot{x}+K_{\mathrm{p}} e=0, \\
& \dot{x}=-L x+K_{\mathrm{d}} e .
\end{aligned}
$$

Consider the Lyapunov function candidate

$$
\begin{aligned}
V_{2}(\dot{q}, e, x)= & \frac{1}{2} \dot{q}^{\mathrm{T}} M(q) \dot{q}+\frac{1}{2} e^{\mathrm{T}} K_{\mathrm{p}} e \\
& +\frac{1}{2}\left(K_{\mathrm{d}} e-L x\right)^{\mathrm{T}}\left(K_{\mathrm{d}} e-L x\right) .
\end{aligned}
$$

The time derivative of $V_{2}(\dot{q}, e, x)$ along the error dynamics (2.7) equals

$\dot{V}_{2}(\dot{q}, e, x)=-\left(K_{\mathrm{d}} e-L x\right)^{\mathrm{T}} L\left(K_{\mathrm{d}} e-L x\right)$,

which, by virtue of $(2.7 b)$, can be written as

$\dot{V}_{2}(\dot{q}, e, x)=-\dot{x}^{\mathrm{T}} L \dot{x}$. 
Equation (2.9) shows that $\dot{V}_{2}(\dot{q}, e, x)$ is only negative-semi-definite. As before, LaSalle's invariance theorem can be applied to complete the proof. To this end, we have to verify that the largest invariant set in

$$
\left\{(\dot{q}, e, x) \mid \dot{V}_{2}(\dot{q}, e, x)=0\right\} \equiv\{(\dot{q}, e, x) \mid \dot{x}=0\}
$$

is the equilibrium state $(\dot{q}, e, x)=0$. Note that

$\dot{x}=0 \rightarrow x=$ constant

and, according to (2.7b), this implies

$e=$ constant .

In addition,

$e=$ constant $\rightarrow \dot{q}=0, \ddot{q}=0$.

Then, from (2.7a), we obtain $e=0$ and, using (2.7b), $x=0$, which implies the thesis.

The gravitation compensating part in the controller is a function of the actual position $q$. From the literature $[15,16]$ it is well known that under a sufficiently strong proportional gain $K_{\mathrm{p}}$, global asymptotic stabilization of the position controller (2.2) is preserved if $G(q)$ is replaced by $G\left(q_{\mathrm{d}}\right)$. It is interesting to notice that the same modification can be implemented in our controller (2.6), as shown below.

Theorem 2.2. Consider the controller

$\tau=G\left(q_{\mathrm{d}}\right)-K_{\mathrm{d}} \dot{x}-K_{\mathrm{p}} e$,

$\dot{x}=-L x+K_{\mathrm{d}} e$,

in closed loop with (2.1). If

$\lambda_{\mathrm{m}}\left(K_{\mathrm{p}}\right)>G_{\mathrm{M}}$,

where $\lambda_{\mathrm{m}}\left(K_{\mathrm{p}}\right)$ represents the smallest eigenvalue of $K_{\mathrm{p}}$, and

$\left\|\frac{\partial G(q)}{\partial q}\right\|<G_{\mathrm{M}}$

with $G_{M}>0$ a constant [16]. Then the equilibrium point $(\dot{q}, e, x) \equiv 0$ is globally asymptotically stable.

Proof. Consider the function

$$
H\left(q, q_{\mathrm{d}}\right)=P(q)-q^{\mathrm{T}} G\left(q_{\mathrm{d}}\right)+\frac{1}{2} q^{\mathrm{T}} K_{\mathrm{p}} q-q^{\mathrm{T}} K_{\mathrm{p}} q_{\mathrm{d}},
$$

where $P(q)$ represents the potential energy of the robot, which is related to the gravitational torque as

$G(q)=\frac{\partial P(q)}{\partial q}$.

In [16] it is shown that $H\left(q, q_{\mathrm{d}}\right)$ has a global minimum at $e \equiv q-q_{\mathrm{d}}=0$ under condition (2.13). Introduce the following Lyapunov function candidate

$$
\begin{aligned}
V_{3}(\dot{q}, e, x)= & \frac{1}{2} \dot{q}^{\mathrm{T}} M(q) \dot{q}+H\left(q, q_{\mathrm{d}}\right)-H\left(q_{\mathrm{d}}, q_{\mathrm{d}}\right) \\
& +\frac{1}{2}\left(K_{\mathrm{d}} e-L x\right)^{\mathrm{T}}\left(K_{\mathrm{d}} e-L x\right),
\end{aligned}
$$

which is positive-definite with respect to $(\dot{q}, e, x)$. The time derivative of (2.17) along the closed-loop dynamics $((2.1)$ and (2.12)) equals

$\dot{V}_{3}(\dot{q}, e, x)=-\dot{x}^{\mathrm{T}} L \dot{x}$.

By invoking LaSalle's invariance theorem the proof can be completed (see also [16]).

\subsection{Discussion}

(1) A comparison of the controllers (2.2) and (2.6) shows that we actually replace the velocity feedback part by a first-order linear compensator. The striking point is that we do not employ the natural state variable $x$ of this compensator in the controller part (2.6a), but its time derivative $\dot{x}$. To motivate this choice, note from (2.6b) that for $K_{\mathrm{d}}=L$ we have

$\ddot{x}=-L(\dot{x}-\dot{q})$.

Owing to the asymptotic convergence of $\ddot{x}$ to zero, (2.19) implies that $\dot{x}$ actually asymptotically reconstructs the velocity signal $\dot{q}$. In terms of classical control theory this linear compensator represents a tame $D$-action.

(2) By rewriting the controller (2.6) as

$\tau=G(q)-y$,

where

$\dot{x}=-L x+K_{\mathrm{d}} e$,

$y=K_{\mathrm{d}} \dot{x}+K_{\mathrm{p}} e=-K_{\mathrm{d}} L x+\left(K_{\mathrm{d}}^{2}+K_{\mathrm{p}}\right) e$.

one can see that the controller consists of a gravitation compensation part and a first-order linear dynamic output feedback part. 
(3) For practical purposes it is useful to know that the controller (2.6) can be generalized as

$\tau=G(q)-K_{\mathrm{d}} \dot{x}-K_{\mathrm{p}} e$,

$\dot{x}=-L_{1} x+L_{2} e$,

where $L_{1}=L_{1}^{\mathrm{T}}>0$ and $L_{2}=L_{2}^{\mathrm{T}}>0$. In comparison to (2.6), this controller does not restrict the choice of $L_{2}$ to $K_{\mathrm{d}}$. Under the assumption that $L_{1}, L_{2}$ and $K_{\mathrm{d}}$ are diagonal, a natural choice in practice, global asymptotic stability of (2.1) and (2.21) can be shown with the Lyapunov function

$$
\begin{aligned}
V_{4}(\dot{q}, e, x)= & \frac{1}{2} \dot{q}^{\mathrm{T}} M(q) \dot{q}+\frac{1}{2} e^{\mathrm{T}} K_{\mathrm{p}} e \\
& +\frac{1}{2}\left(L_{2} e-L_{1} x\right)^{\mathrm{T}} K\left(L_{2} e-L_{1} x\right)
\end{aligned}
$$

where $K=L_{2}^{-1} K_{\mathrm{d}}>0$ is diagonal. The proof is analogous to the proof of Theorem 2.1. It will be clear that the same generalization holds for the desired gravitation compensating control law (2.12).

\section{Simulation results}

The performance of the controller (2.6) was studied by simulation. To this end, a two-degreesof-freedom robot manipulator moving in the vertical plane was considered, see Figure 1 [9]. The dynamics of this system satisfy (2.1), where $M(q), C(q, \dot{q}), G(q)$ are given in the Appendix.

In these simulations, performed with SIMULAB ${ }^{\mathrm{TM}}$, the robot system is required to move to the desired set point $q_{\mathrm{d}}^{\mathrm{T}}=\left[\begin{array}{ll}1 & 1\end{array}\right]$. The initial con-

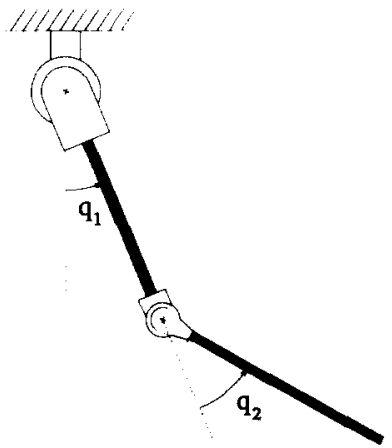

Fig. 1. Two DOF robot system [9]

ditions for $q, \dot{q}$ and $x$ are assumed to be equal to zero. The controller gain matrices satisfy

$$
\begin{gathered}
K_{\mathrm{p}}=\left[\begin{array}{cc}
50 & 0 \\
0 & 100
\end{array}\right], \quad K_{\mathrm{d}}=\left[\begin{array}{cc}
150 & 0 \\
0 & 60
\end{array}\right], \\
L=\left[\begin{array}{cc}
80 & 0 \\
0 & 100
\end{array}\right] .
\end{gathered}
$$

The results are depicted in Figure 2. From this figure it can be concluded that the robot system moves reasonably fast initially towards its desired endpoint, but the final positioning at this endpoint requires more time. This is apparently caused by the presence of the dynamic compensator, which represents a kind of integral action, for which it is well known that it generally slows down the stabilization process.
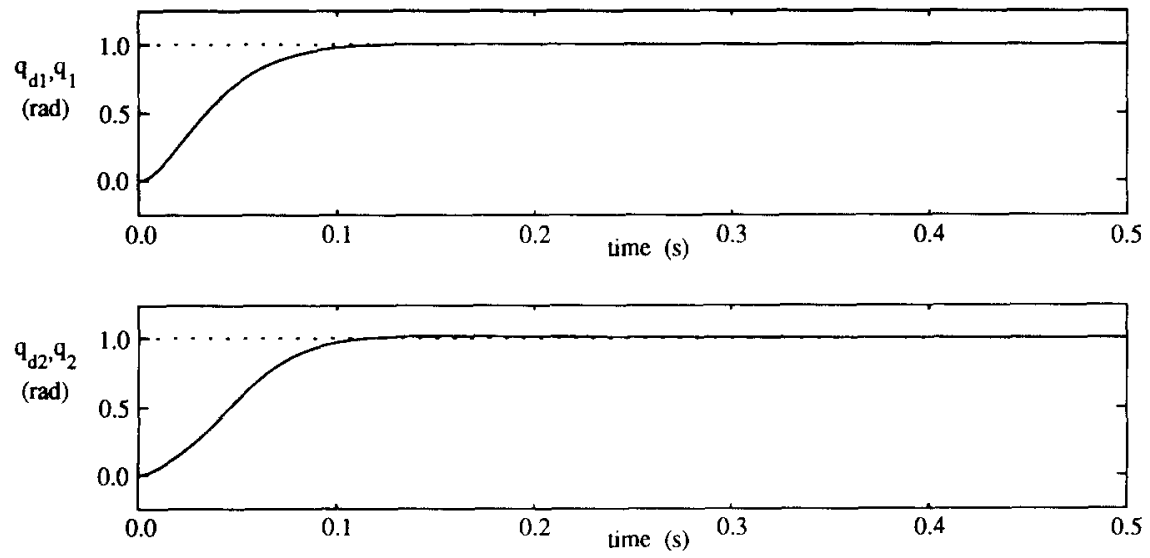

Fig. 2. Desired and actual positions 


\section{Conclusions}

A computationally efficient position controller for robots is presented that requires only joint position measurements. The controller consists of a gravitation compensation and a linear dynamic first-order compensator. It was shown that the gravitation compensation part can be implemented in two ways, either by exact gravitation compensation or by desired gravitation compensation. For both solutions global asymptotic stability is established by invoking LaSalle's invariance theorem. In a simulation study of a two-degrees-of-freedom robot system, the performance of the presented controller was illustrated.

We would like to stress that the presented controllers are especially interesting for industrial purposes. As is well known, an important design constraint in the development of robotic systems is their economic feasibility. The stabilizing position controllers presented in this note replace the need for expensive velocity acquisition hardware by a linear first-order compensator, which can easily be realized in software. This underscores the attractivity of the controllers.

\section{Acknowledgements}

The first author would like to thank The Netherlands' Technology Foundation (STW) for supporting his work. We also gratefully acknowledge Dr. Rafael Kelly (Ensenada, Mexico) who sent us, after submission of this note, the manuscript [7] in which a similar result has been obtained.

\section{Appendix}

The robot system is characterized by [9]

$$
M(q)=\left[\begin{array}{cc}
8.77+1.02 \cos \left(q_{2}\right) & 0.76+0.51 \cos \left(q_{2}\right) \\
0.76+0.51 \cos \left(q_{2}\right) & 0.62
\end{array}\right],
$$

$C(q, \dot{q})=\left[\begin{array}{cc}-0.51 \sin \left(q_{2}\right) \dot{q}_{2} & -0.51 \sin \left(q_{2}\right)\left(\dot{q}_{1}+\dot{q}_{2}\right) \\ 0.51 \sin \left(q_{2}\right) \dot{q}_{1} & 0\end{array}\right]$,
$G(q)=g\left[\begin{array}{c}7.6 \sin \left(q_{1}\right)+0.63 \sin \left(q_{1}+q_{2}\right) \\ 0.63 \sin \left(q_{1}+q_{2}\right)\end{array}\right]$,

where $g$ is the acceleration of gravity.

\section{References}

[1] P.R. Bélanger, Estimation of angular velocity and acceleration from shaft encoder measurements, in: Proc. IEEE Conf. on Robotics and Automation, Nice, France (1992) 585-592.

[2] H. Berghuis and H. Nijmeijer, Robust control of robots using only position measurements, Memorandum no. 1073 (1992), Dept. of Applied Mathematics, Univ. of Twente, Enschede, Netherlands; accepted for IFAC World Congress 1993, Sydney, Australia.

[3] H. Berghuis, H. Nijmeijer and P. Löhnberg, Observer design in the tracking control problem of robots, in: Proc. IFAC Symp. NOLCOS '92, Bordeaux, France (1992) 588-593.

[4] C. Canudas de Wit and N. Fixot, Robot control via robust estimated state feedback, IEEE Trans. Automat. Control 36 (1991) 1497-1501.

[5] C. Canudas de Wit, N. Fixot and K.J. Åström, Trajectory tracking in robot manipulators via nonlinear estimated state feedback, IEEE Trans. Robotics Automat. 8 (1992) $138-144$.

[6] W. Hahn, Stability of Motion (Springer, Berlin, 1967).

[7] R. Kelly, A simple set-point robot controller by using only position measurements, Internal Report, Div. de Fisica Aplicada, CICESE, Ensenada, Mexico, 1992, accepted for IFAC World Congress 1993, Sydney, Australia.

[8] R.D. Klafter, T.A. Chmielewski and M. Negin, Robotic Engineering - An Integrated Approach (Prentice-Hall, Englewood Cliffs, 1989).

[9] L. Kruise, Modelling and control of a flexible beam and robot arm, Ph.D. Dissertation, Univ. of Twente, Enschede, Netherlands, 1990.

[10] S. Nicosia and P. Tomei, Robot control by using only joint position measurements, IEEE Trans. Automat. Control 35 (1990) 1058-1061.

[11] S. Nicosia, A. Tornambé and P. Valigi, Experimental results in state estimation of industrial robots, in: Proc. Conf. on Decision and Control, Honolulu (1990) 360-365.

[12] R. Ortega and M.W. Spong, Adaptive motion control of rigid robots: a tutorial, Automatica 25 (1989) 877-888.

[13] A. van der Schaft, System theory and mechanics, in: H. Nijmeijer and J.M. Schumacher, Eds., Three Decades of Mathematical System Theory, Lecture Notes in Control and Information Sciences. Vol. 135 (Springer, Berlin, 1990) $426-452$.

[14] M.W. Spong and M. Vidyasagar, Robot Dynamics and Control (Wiley, New York, 1989).

[15] M. Takegaki and S. Arimoto, A new feedback method for dynamic control of manipulators, ASME J. Dynamic Systems, Measurement, and Control 102 (1981) 119-125.

[16] P. Tomei, Adaptive PD controller for robot manipulators, IEEE Trans. Robotics Automation 7 (1991) 565-570. 